\title{
An Approach to Interdisciplinary Orthodontics
}

\author{
Manash Jyoti Borah \\ Consultant Oral and Dental Surgeon, Beena Medical Hall, D.K. Road, \\ North Lakhimpur, Lakhimpur, Assam, Pin:787001
}

\begin{abstract}
Patient seeking orthodontic treatment in the present era comprises of both children and adults, thereby increasing the importance of multidisciplinary approach in orthodontics immensely. The interdisciplinary approach requires active involvement of various specialists like Prosthodontist, Oral and maxillofacial Surgeon, ENT surgeon, Paediatrician, Pedodontist, Endodontist, Periodontist, Audiologist, Physiotherapist etc. A combined interdisciplinary treatment approach will yield a result best suited for the patient as well as clinicians. Orthodontist in such Interdisciplinary treatment approach can play a primary or secondary role. So, the purpose of this article is to briefly summarize interdisciplinary approach for the management of dentofacial problems.
\end{abstract}

Keywords: Interdisciplinary Orthodontics, Orthodontist, multidisciplinary approach in orthodontics

\section{INTRODUCTION}

Interdisciplinary approach is of vital importance for patients with mutilated dentition. Mutilated dentition is often seen in patients who have had dental diseases and often did not receive any form of preventive or interceptive therapy. As a result, their teeth are often malposed, periodontally compromised, extensively worn or abraded and poorly restored. Patients with congenital defects also benefit from interdisciplinary care as it idealizes the dentition to an esthetically and functional acceptable result. [1]

Adult orthodontics has gained its pace in late 1970s, to become a common therapy today. Treatment procedures differs in adolescents, children and adults. In adults the other types of dental treatment almost always are required, which makes interdisciplinary approach an important aspect in orthodontics. Because absence of growth in adults makes growth modification treatment objective an obsolete one. Everything has to be done with either tooth movement, restorative dentistry or orthognathic surgery. [2]

The role of orthodontist in the Interdisciplinary Orthodontics Therapy (IDT) may be either primary or secondary. Primary as in case where orthodontic patient requires involvement of allied specialties treatment as prosthetic replacement of missing teeth, periodontal rehabilitation, surgical exposure of an impacted tooth etc. Secondary as in case of space creation or tooth uprighting to facilitate prosthetic replacement of a missing tooth etc.

Conditions requiring Inter-disciplinary care:

1. Missing tooth /Space Management

$$
\text { i. Tooth agenesis }
$$

ii. Extracted teeth

2. Malformed teeth

i. $\quad$ Single malformed teeth

ii. Multiple malformed teeth [1]

3. Fractured tooth

4. Periodontally compromised dentition

5. Trauma from occlusion

6. Congenital defects

7. Transpositioned Teeth

8. Gingival discrepancies

i. Anterior aesthetic zone

ii. Vertical maxillary excess or jaw deficiency

9. Severe jaw discrepancies 


\section{Missing Teeth/Space Management}

Most commonly congenitally missing teeth reported is the mandibular second premolars followed by lateral incisors, except the third molars.[3] Primary second molars in absence of mandibular second premolars can be maintained for a long term.[4] If they are submerged due to ankylosis, they can be restored to the functional occlusal plane with restorations such as occlusal composites. However, if

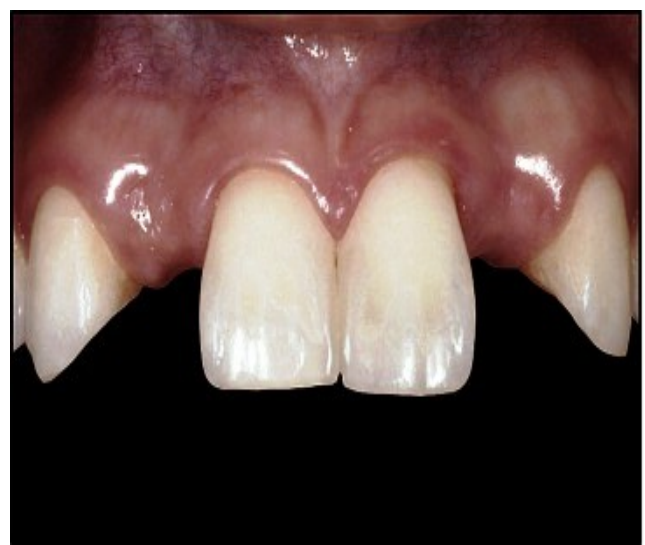

Figure 1: Congenitally missing Lateral incisor and replaced by prosthesis

Absence of lateral incisors can be commonly treated by:

a. Space Closure

b. Opening up of spaces for prosthetic rehabilitation.

\section{a. Space closure}

Space closure is done initially followed by esthetic reshaping of the canine to lateral incisor. In patient having high smile line, the canine may require slight extrusion to establish an ideal gingival relationship between the central incisor and canine in the lateral incisor site. To give an ideal shade to a darker shade canine, bleaching or veneering of the canine may be required.

b. Opening up of spaces for the Prosthetic replacement of missing lateral

It can be done by distalising the canine into class I relationship with the opposing canine. Spear FM (1997) has opted for the ideal replacement for the missing teeth to be a dental implant provided all factors are favorable. The amount of space required for the placement of implant is predetermined the neighbouring teeth have drifted and tipped over the occlusal level of the primary molar, orthodontic intervention may be necessary to reposition the teeth.

Congenitally missing lateral incisor presents spacing in the anterior aesthetic zone. Lateral incisor guides the eruption of the permanent canine to its ideal position in the dental arch. Due to lack of eruption guidance, impaction and ectopic eruption can occur.[1]

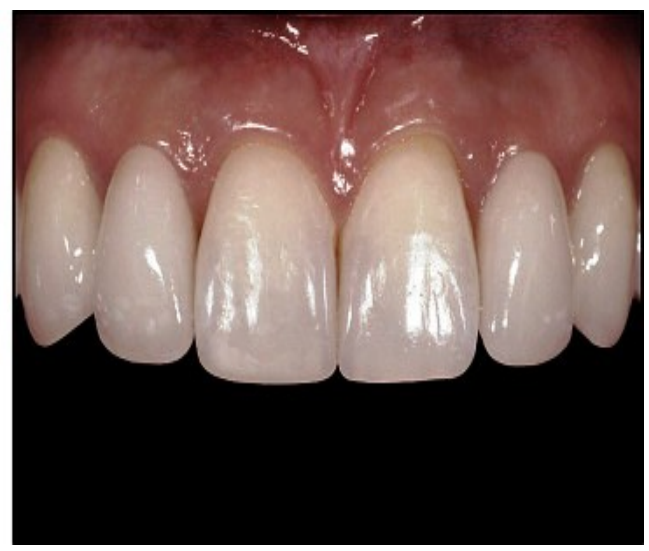

and created accordingly with orthodontic treatment. Space evaluation is of importance especially between the roots and the level of the crowns to ensure successful crown placement. Implant placement is done once the vertical growth of the patient is completed, usually 14-15 years of age in girls and 16-17 years of age in boys.[6] Prosthetic replacement like Maryland bridge or a pontic on a retainer can be used according to the convenience for the patient.

\section{Extraction}

Due to certain dental diseases like the dental caries, periodontal disease or traumas the patient loses teeth. If the edentulous period is extensive it results in shifting and tipping of the individual dental units resulting in alteration of equilibrium of the dentition.

The proposed treatment protocols are:

a. Orthodontic closure of the edentulous space.

b. Prosthetic Replacement:

Tipped teeth may be included in the design of partial prosthesis but tipped teeth 
are often not best fit for abutment for fixed or removable prosthesis as the occlusal forces are not along the long axis of tipped teeth and cause three wall bony defects on the side of the tip. Also supreruption of teeth in opposing arch can interfere with an ideal prosthetic replacement of missing teeth. The proposed treatment includes both orthodontic and restorative treatment. The axial inclination of the tipped teeth and intrusion of the antagonistic supraerupted teeth is corrected orthodontically which helps to improve the periodontal prognosis as well as occlusion.[1]

\section{Teeth Malformation a. Single Teeth}

In the esthetic anterior zone of the mouth, the most common malformations seen is the peg shaped lateral incisors. It creates uneven space distribution, a midline deviation and disturbed occlusion. Fixed orthodontic mechanotherapy with space management and bite correction can be employed to manage such discrepancies. Restorative reconstruction of the shape and size of the malformed teeth with the help of ceramic crown and veneers is done. If full coverage restoration is planned the over jet can be selectively increased by $0.5-075 \mathrm{~mm}$ on the peg lateral to minimize lingual reduction. The overbite is kept minimal in cases when veneers are planned in the restorative treatment.[1]

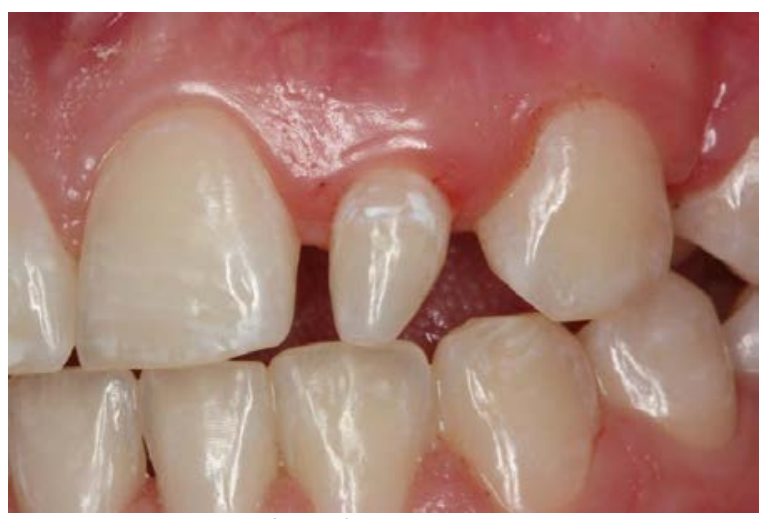

Figure 2: Peg Lateral

\section{b. Multiple Teeth}

Multiple teeth malformation are relatively uncommon and such patients may have other systemic conditions which need to be addressed during treatment planning. A comprehensive diagnosis is must. The role of orthodontist in such case is to facilitate any subsequent restorative care through space management and bite correction.

\section{Fractured Tooth}

Teeth fractured above the gingival margin can be readily restored with a cast or prefabricated post. However, if teeth is fractured below the gingival margin, it may encroach on the biological width and can create a significant restorative challenges. Fractured teeth below the gingival margin have following treatment options:

i. Extraction of the fractured tooth, followed by prosthetic replacement.

ii. Periodontal surgery to reposition the gingival margin to an appropriate level to expose the sound tooth structure for restorative reconstruction of tooth.[7]

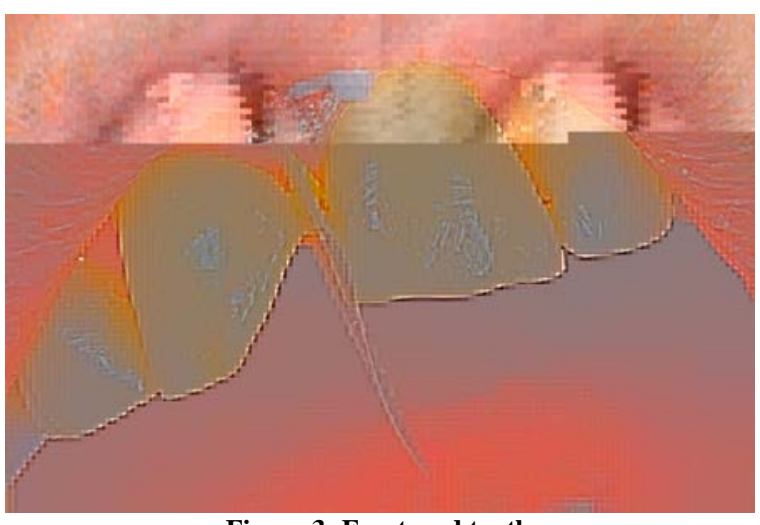

Figure 3: Fractured tooth

\section{Periodontal Compromised Dentition}

The interrelationship between orthodontics and periodontics is a symbiotic relationship. Periodontal condition which requires orthodontic treatment includesmidline diastema and correction of black triangles. Adult patients previously affected by periodontal disease often present with "Black Triangles", due to missed interdental papillae height. By means of orthodontic mechanotherapy it is possible to correct teeth position and improve soft tissue aesthetics. Various pathological migration such as intrusion, extrusion, rotation and uprighting are needed to achieve an esthetically acceptable outcome. 
Localised, aggressive periodontitis is characterized by localized breakdown of the periodontal attachment in particular parts of the dental arch in life [8]. An interdisciplinary approach combining of regenerative periodontal therapy, prosthodontic rehabilitation and orthodontic treatment is necessary to treat a patient with pathologic migration of teeth due to localized aggressive periodontitis.

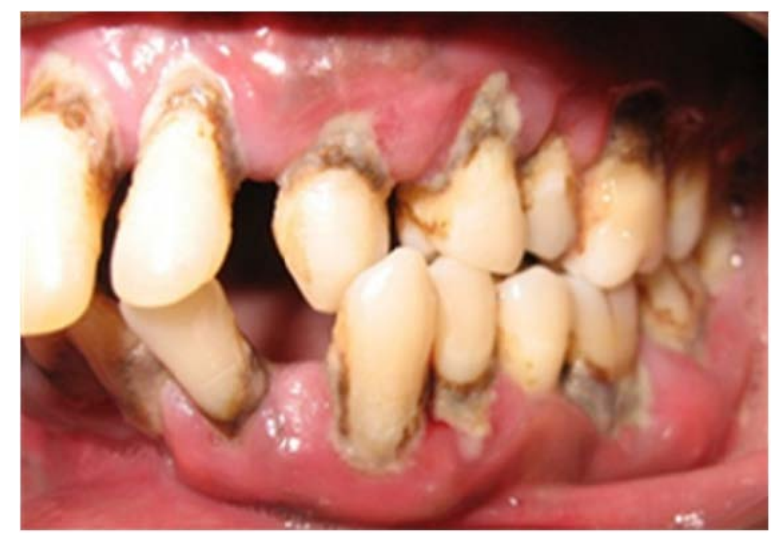

Figure 4: Periodontal Compromised Dentition

\section{Trauma from occlusion}

When the occlusal force exceeds the adaptive capacity of the tissues, tissue injury occurs. The resultant injury is called trauma from occlusion. It may be primary or secondary trauma from occlusion.

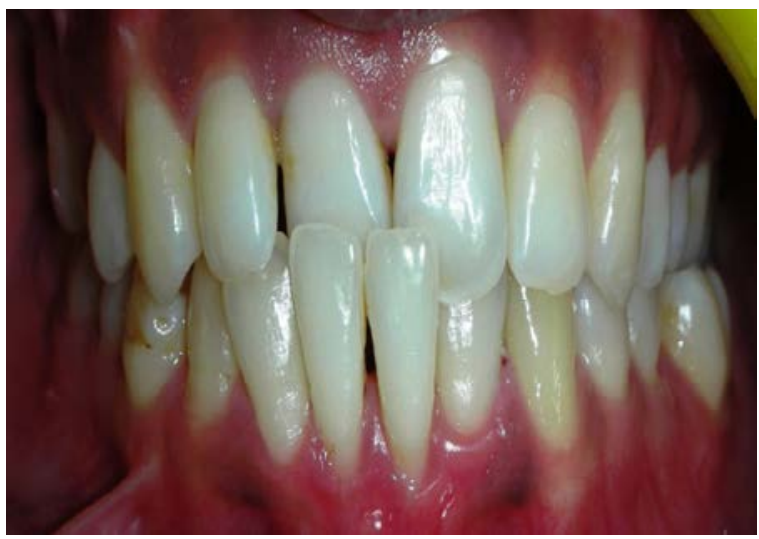

Figure 5: Trauma from Occlusion

Reversible Approach includes management of parafunctional habits through patients' education and the use of occlusal splints.

Irreversible approach includes:

i. Selective grinding

ii. Temporary or provisional splinting of mobile teeth. iii. Orthodontic therapy

iv. Occlusal reconstruction

v. Extraction of selected teeth.

\section{Congenital Defects}

Cleft lip and palate are a congenital problem affecting around 1 in 1000 births globally. Cleft lip and palate impair the development of teeth, speech, hearing along with associated esthetic and psychological problems.

Cleft lip and palate are managed by a group of specialists consisting of an Orthodontist, Oral surgeon, ENT Surgeon, Audiologist, Neurologist, Speech Therapist, Geneticist and Pediatrician.

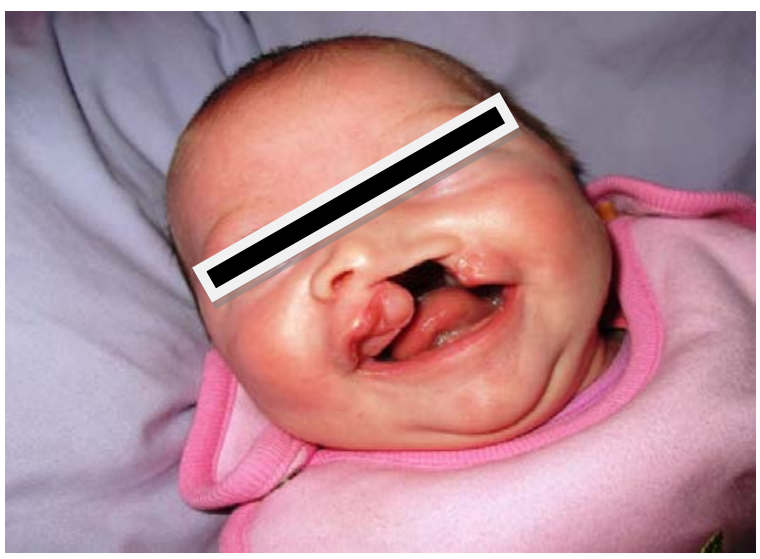

Figure 6: Cleft Lip and Palate

\section{Transplantation of Tooth}

Transmigration refers to the physiological migration of an unerupted tooth across the midline in the absence of pathology or trauma. Multidisciplinary care involves orthodontic creation of space, surgical extraction of the transmigrated tooth followed by implant replacement and crown or autotransplantation of transmigrated tooth to the recipient site.

\section{Surgical Exposure of Impacted Tooth}

Third molars are the most impacted teeth followed by maxillary canines, second premolars, maxillary central incisors and mandibular canines. In orthodontics, the maxillary canines are the most frequently surgically exposed teeth for alignment and leveling in the dental arch. A team approach is required to provide an excellent esthetic and functional result. 


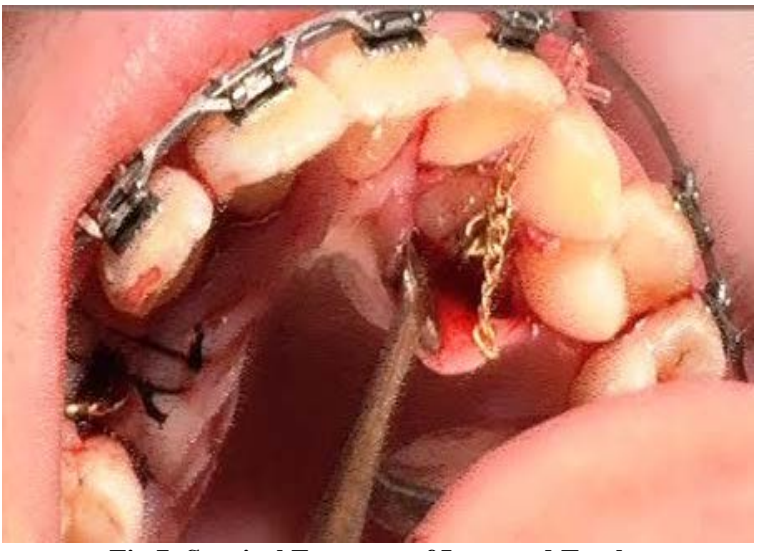

Fig 7: Surgical Exposure of Impacted Tooth:

\section{Mucogingival Discrepancies}

Ideally prosthodontic treatment is carried out to correct following, prior to orthodontic mechanotherapy-

a. Height of the gingival margins.

b. Contour of the labial gingival margins.

c. Embrasure corrections.

Management includes reshaping of interproximal areas of teeth crowns followed by orthodontic space closure or orthodontic correction of root angulation or periodontal regeneration therapy.

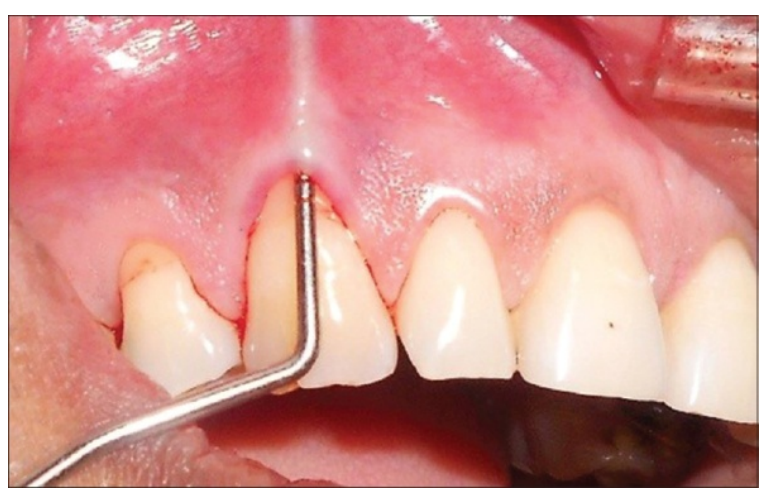

Figure 8: Mucogingival discrepancies.

\section{Severe Jaw Discrepancies}

Certain conditions like congenital anomalies excessive large or small jaw, marked asymmetric jaw growth etc. requires surgical intervention along with orthodontic mechanotherapy. Carried out with oral and maxillofacial Surgeon, certain jaw surgeries like sagittal split osteotomy, Le fort surgery, Subapical surgery, Chin Surgeries etc. are carried out to correct the dysplasia.

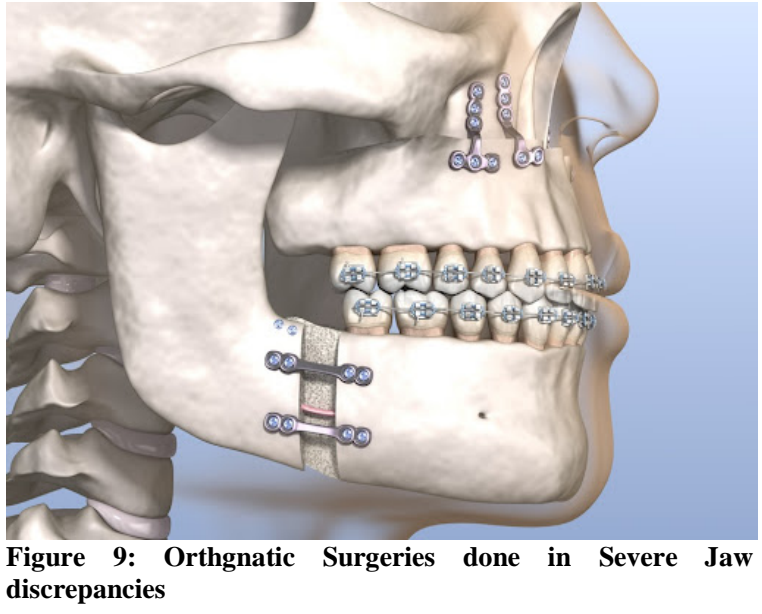

\section{CONCLUSION}

Orthodontics and dentistry as a whole are a dynamic branch of medical science. In certain situation dental treatment demands a multidisciplinary approach to manage certain oral and dentofacial manifestations. A thorough diagnosis, with a clear treatment objective with a sound treatment plan is required to manage those conditions. A team approach with specialist of allied medical and dental specialities is required to meet the needs of the patients.

\section{Acknowledgement: None}

\section{Conflict of Interest: None}

\section{Source of Funding: None}

\section{REFERENCES}

1. Kharbanda O.P. Orthodontics: Diagnosis and Management of Malocclusion and Dentofacial Deformities $3^{\text {rd }}$ Edition; 2020.

2. Profitt WR, Fields HW, Sarver DM. Contemporary Orthodontics, $5^{\text {th }} \quad$ edition, Elsevier; 2017

3. Vastardis H. The genetics of human tooth agenesis: new discoveries for understanding dental anomalies. Am J Orthod Dentofac Orthop 2000; 117:650-56

4. Kokich V.G, Spear F.M. Guidelines for managing the orthodontic-restorative patient. Semin Orthod 1997; 3:3-20

5. Heij DG, OPdebeeck H, Van Steenberghe D, Kokich VG, Belser U, Quirynen M.Facial development, continuous tooth eruption and mesial drift as compromising factors for implant placement. Int $\mathrm{J}$ Oral Maxillofac Implants 2006; 21:867-78 
6. Eissmann HF, Radke RA, Noble WH. Physiologic design criteria for fixed dental restorations. Dental Clin North Am 1971; 15:543-68

7. Reed BE, Polson AM, Subtelny JD. Long term periodontal status of teeth moved into extraction sites. Am J Orthod Dentofac Orthop 1985; 88:203-8
8. Rohrer A. Displaced and impacted canines. Int J Orthod Oral Surg 1929; 15:1002-1004

How to cite this article: Manash Jyoti Borah. An approach to interdisciplinary orthodontics. International Journal of Science \& Healthcare Research. 2021; 6(4): 205-210. DOI: https:// doi.org/10.52403/ijshr.20211029 